PROCEEDINGS OF THE

AMERICAN MATHEMATICAL SOCIETY

Volume 130, Number 1, Pages 15-21

S 0002-9939(01)05983-4

Article electronically published on April 26, 2001

\title{
A NOTE ON THE NAKAI CONJECTURE
}

\author{
PAULO BRUMATTI, YVES LEQUAIN, DANIEL LEVCOVITZ, AND ARON SIMIS
}

(Communicated by Wolmer V. Vasconcelos)

\begin{abstract}
The conjectures of Zariski-Lipman and of Nakai are still open in general in the class of rings essentially of finite type over a field of characteristic zero. However, they have long been known to be true in dimension one. Here we give counterexamples to both conjectures in the class of onedimensional pseudo-geometric local domains that contain a field of characteristic zero. Likewise, in connection with a recent result of Traves on the Nakai conjecture, we also show that their hypothesis of finite generation of the integral closure cannot be removed even in the class of local domains containing a field of characteristic zero.
\end{abstract}

\section{INTRODUCTION}

Let $A$ be a commutative ring and let $k \subset A$ be a subring. The set $\operatorname{End}_{k}(A)$ of $k$-module endomorphisms of $A$ is an $A$-module in the usual way whereby scalar multiplication $a \rho\left(a \in A, \rho \in \operatorname{End}_{k}(A)\right)$ is to be taken as the composite $\mu_{a} \circ \rho$, with $\mu_{a}$ denoting multiplication by $a$. Given an integer $r \geq 0$, let $\operatorname{Diff}_{k}^{r}(A) \subset \operatorname{End}_{k}(A)$ denote the $A$-submodule consisting of the differential operators of order $\leq r$ of the $k$ algebra $A$. The traditional reference for differential operators of algebras is 3 . One has $\operatorname{Diff}_{k}^{0}(A)=A$ and $\operatorname{Diff}_{k}^{r}(A)=A \oplus \operatorname{Der}_{k}^{r}(A)$ for every $r \geq 1$, where $\operatorname{Der}_{k}^{r}(A)$ is the module of (higher) derivations of order $\leq r$ of $A$ as introduced in 8 . For many a purpose the following submodule is sufficient. Namely, one lets $\operatorname{der}_{k}^{r}(A) \subset \operatorname{Der}_{k}^{r}(A)$ stand for the $A$-submodule generated by the composites of $r$ ordinary derivations (i.e., elements of $\operatorname{Der}_{k}^{1}(A)$ ). Clearly, $\operatorname{der}_{k}^{1}(A)=\operatorname{Der}_{k}^{1}(A)=\operatorname{der}_{k}(A)$, which is isomorphic to the $A$-dual of the $A$-module of Kähler differentials $\Omega_{k}(A)$. One of the earliest results was to the effect that if $A$ is a regular ring essentially of finite type over a field $k$, then $\Omega_{k}(A)$ (hence also $\operatorname{der}_{k}(A)$ ) is $A$-projective and, provided $k$ has characteristic zero, the equality $\operatorname{der}_{k}^{r}(A)=\operatorname{Der}_{k}^{r}(A)$ holds for all $r \geq 1$.

Various converse-like questions can be naturally posed, the most celebrated of which are:

(1) (The Nakai conjecture) If $A$ is a ring essentially of finite type over a field $k$ of characteristic zero such that $\operatorname{der}_{k}^{r}(A)=\operatorname{Der}_{k}^{r}(A)$ for all $r \geq 1$, then $A$ is regular.

Received by the editors January 11, 2000 and, in revised form, May 19, 2000.

2000 Mathematics Subject Classification. Primary 13M05, 13M10, 13N15, 13B22; Secondary 14F10, 12H05, 13B25, 13F25.

Key words and phrases. Derivations, integral closure, pseudo-geometric, $\mathcal{D}$-stable.

The authors were partially supported by $\mathrm{CNPq}$ and by a grant from the group ALGAPRONEX/MCT. 
(2) (The Zariski-Lipman conjecture) If $A$ is a ring essentially of finite type over a field $k$ of characteristic zero such that $\operatorname{der}_{k}(A)$ is $A$-projective, then $A$ is regular.

These conjectures have an interesting history and have been verified for several classes of rings over a field $k$ of characteristic zero. For example, it has been shown by Mount and Villamayor ([7]) that Nakai's conjecture is true in dimension one. On the other hand, Lipman noted quite early (cf. 4]) that under the assumption of the Zariski-Lipman conjecture the ring $A$ is at least normal. In the same vein, Traves has recently proved that if $A$ is a local domain satisfying the equalities $\operatorname{der}_{k}^{r}(A)=\operatorname{Der}_{k}^{r}(A)$ for all $r \geq 1$, such that its integral closure $A^{\prime}$ is a finite $A$ module and $\operatorname{der}_{k}\left(A^{\prime}\right)$-simple, then $A$ is normal (cf. [11]). A consequence of this, as he observed, is a new proof of Nakai's conjecture in dimension one.

In this paper one first looks at the feasibility of extending the Nakai conjecture beyond the class of rings $A$ whose integral closure is not a finite $A$-module. The main result here is an example showing that such an extension is not possible even for rings of dimension one. The example itself is due to Goodearl and Lenagan ([2]), and later reformulated by Maloo ([5]) in a way that will be convenient for our purpose. This example was originally looked upon from a slightly different perspective. Here we consider it from the viewpoint of higher derivations and the Nakai conjecture. As it turns out, there will be quite a few nontrivial things to be proved.

Then one considers the possibility of extending the realm of Nakai's conjecture to the class of one-dimensional pseudo-geometric rings. Here too one shows, by means of an example, that such an extension is not possible. The same example is also suited to show that the Zariski-Lipman conjecture cannot be extended to a larger class of rings that include the one-dimensional pseudo-geometric rings.

\section{The FIRST EXAMPLE}

We recall Maloo's description of the Goodearl-Lenagan local domain.

Let $k$ be a field of characteristic zero and let $Y=\exp (X)=\sum_{n \geq 0} X^{n} / n ! \in$ $k[[X]]$. Since $k(X, Y)$ is a purely transcendental extension of $k$ of transcendence degree 2 , one has the ordinary partial derivatives $\partial_{X}, \partial_{Y} \in \operatorname{der}_{k}(k(X, Y))$.

Set $B=k[[X]] \cap k(X, Y)$. Clearly, $B$ is a rank one discrete valuation ring with $B[1 / X]=k(X, Y)$ and maximal ideal generated by $X$ (or $Y-1$ ). Let $d_{X} \in$ $\operatorname{der}_{k}(k[[X]])$ be the unique $k$-derivation sending $X^{i}$ to $i X^{i-1}$. Since $d_{X}(X)=1$ and $d_{X}(Y)=d_{X}(\exp (X))=\exp (X)=Y \in k[X, Y], d_{X}$ induces a $k$-derivation of the polynomial ring $k[X, Y]$, hence also a $k$-derivation $d$ of its field of fractions $k(X, Y)$.

One easily sees that $d(B) \subset B$ (hence $\left.d \in \operatorname{der}_{k}(B)\right)$ and that $d=\partial_{X}+Y \partial_{Y}$. Set $A=\left\{b \in B \mid \partial_{Y}(b) \in B\right\}$. Obviously, $k[X, Y] \subset A$ and $\partial_{Y}(A) \subset B$. As in [5, Example], one can show that $A$ is a nonnormal one-dimensional local domain whose integral closure $A^{\prime}$ is $B$.

Recall that, quite generally, if $B$ is a $k$-algebra and if

$$
\mathcal{D} \subset \operatorname{Der}_{k}^{\infty}(B)=\bigcup_{n \geq 0} \operatorname{Der}_{k}^{n}(B),
$$

then $B$ is said to be $\mathcal{D}$-simple if for every nonzero ideal $I$ of $B$ there exists $d \in \mathcal{D}$ such that $d(I) \not \subset I$. As the minimal prime ideals of a $\mathcal{D}$-stable ideal are $\mathcal{D}$-stable, 
this definition is equivalent to the following: for every nonzero prime ideal $\wp$ of $B$, there exists $d \in D$ such that $d(\wp) \not \subset \wp$.

Our result in this section is the following.

(1.1) Theorem. With the above notation, $A$ is a one-dimensional local domain with integral closure $A^{\prime}$ satisfying the following conditions:

(i) $A^{\prime}$ is $\operatorname{Der}_{k}^{\infty}\left(A^{\prime}\right)$-simple.

(ii) $A^{\prime}$ is not a finite $A$-module.

(iii) $\operatorname{der}_{k}^{n}(A)=\operatorname{Der}_{k}^{n}(A)$ for every $n \geq 0$.

Proof. (i) Since $A^{\prime}$ is a discrete valuation ring, its maximal ideal is the only nonzero prime ideal of $A^{\prime}$. On the other hand, this ideal is generated by $X$ and we have seen that $d$ is a $k$-derivation of $A^{\prime}$ with $d(X)=1$. Therefore, $A^{\prime}$ is $\{d\}$-simple, hence certainly $\operatorname{Der}_{k}^{\infty}\left(A^{\prime}\right)$-simple.

(ii) We claim that the $k$-derivation $d \in \operatorname{der}_{k}(B)$ restricts to a $k$-derivation of $\operatorname{der}_{k}(A)$. Indeed, one has $\left[\partial_{Y}, d\right]=\partial_{Y}$ as $k$-derivations of $k(X, Y)$ since they attain the same values on the field generators. $\partial_{Y}(d(a))=\left[\partial_{Y}, d\right](a)+d\left(\partial_{Y}(a)\right)=\partial_{Y}(a)+$ $d\left(\partial_{Y}(a)\right) \in B$, hence $d(a) \in A$ by definition. On the other hand, the proof of (i) actually showed that $A$ is $\operatorname{der}_{k}(A)$-simple. By [10, $A^{\prime}$ is not a finite $A$-module.

(iii) This is by far the main bulk of the theorem. By the proof of (ii), $d \in \operatorname{der}_{k}(A)$. The assertion will then follow from the following result which may have independent interest.

(1.2) Proposition. Keeping the above notation, let $\Delta \in \operatorname{der}_{k}(A)$ denote the restriction of $d$ to $A$. Then, for every $n \geq 1, \operatorname{Der}_{k}^{n}(A)$ is a free $A$-module with basis $\left\{\Delta, \Delta^{2}, \ldots, \Delta^{n}\right\}$.

For the proof one uses the following technical lemmas, keeping the above notation throughout.

(1.3) Lemma. For any integers $i, j \geq 0, m \geq 1$, the following equalities hold in $B=A^{\prime}$ :

$$
\left[\cdots \left[[d^{i} \circ \partial_{Y}^{j}, \underbrace{X], X], \ldots, X}_{m \text { times }}]= \begin{cases}i(i-1) \cdots(i-m+1) d^{i-m} \circ \partial_{Y}^{j} & \text { if } i>m, \\ i ! \partial_{Y}{ }^{j} & \text { if } i=m, \\ 0 & \text { if } i<m .\end{cases}\right.\right.
$$

Proof. Since $\left[\partial_{Y}, X\right]=\partial_{Y}(X)=0$, it follows that

$$
\left[\ldots \left[[d^{i} \circ \partial_{Y}^{j}, \underbrace{X], X], \ldots, X}_{m \text { times }}]=\left[\ldots \left[[d^{i}, \underbrace{X], X], \ldots, X}_{m \text { times }}] \circ \partial_{Y}^{j} .\right.\right.\right.\right.
$$

As $d(X)=1$, then $\left[d^{i}, X\right]=i d^{i-1}$.

Now apply induction on $m$.

(1.4) Lemma. The set $\left\{d^{i} \circ \partial_{Y}^{j}, 0 \leq i, j, 1 \leq i+j \leq n\right\}$ is a basis of the $k(X, Y)$ vector space $\operatorname{Der}_{k}^{n}(k(X, Y))$. Furthermore, given $D \in \operatorname{Der}_{k}^{n}(k(X, Y))$, if one writes $D=E+F$ with $E \in \sum_{i=1}^{n} k(X, Y) d^{i}$ and $F \in \sum_{j \geq 1} k(X, Y) d^{i} \circ \partial_{Y}^{j}$, then:

(a) $D(A) \subset A \Rightarrow E \in \sum_{i=1}^{n} A d^{i}$ and $F(A) \subset A$,

(b) $F(A) \subset A$ and $F \in \sum_{i=1}^{n} k(X, Y) \partial_{Y}^{j} \Rightarrow F=0$.

Proof. The first result involves an elementary transformation of the canonical basis $\left\{\partial_{X}^{i} \circ \partial_{Y}^{j}, 0 \leq i, j, 1 \leq i+j \leq n\right\}$ of $\operatorname{Der}_{k}^{n}(k(X, Y))=\operatorname{der}_{k}^{n}(k(X, Y))$. In fact, as 
we have seen before, $\partial_{X}=d-Y \partial_{Y}$. Note the semi-commutation rule $\partial_{Y}^{j} \circ d^{j}=$ $(i \mathfrak{I}+d)^{j} \circ \partial_{Y}^{j}$ (where $\mathfrak{I}$ denotes the identity operator), which follows by a direct computation:

$$
\begin{aligned}
\partial_{Y}^{i} \circ d^{j} & =\partial_{Y}^{i} \circ\left(\partial_{X}+Y \partial_{Y}\right)^{j} \\
& =\partial_{Y}^{i} \circ\left(\sum_{k=0}^{j}\left(\begin{array}{l}
j \\
k
\end{array}\right) \partial_{X}^{k}\left(Y \partial_{Y}\right)^{j-k}\right. \\
& =\left(\sum_{k=0}^{j}\left(\begin{array}{l}
j \\
k
\end{array}\right) \partial_{X}^{k}\left(Y \partial_{Y}+i \mathfrak{I}\right)^{j-k}\right) \circ \partial_{Y}^{i} \\
& =(d+i \mathfrak{I})^{j} \circ \partial_{Y}^{i} .
\end{aligned}
$$

Then, a calculation using this rule yields that the vectors of the canonical basis are $k(X, Y)$-linear combinations of vectors of the proposed set. Since the two sets have the same number of elements, one is through.

So, now let $D=E+F$ be as prescribed in the statement of the lemma. In order to prove (a), write $E=\sum_{i=1}^{n} h_{i} d^{i}$. Since $X \in A$ then $h_{1}=D(X) \in A$. A recursion applying $D$ to the successive powers of $X$ yields $h_{i} \in A$ for $i=1, \ldots, n$. Since $d$ restricts to $A$, one gets $E(A) \subset A$ and, consequently, $F(A) \subset A$.

In order to prove assertion (b), write $F=\sum_{j=1}^{n} h_{j} \partial_{Y}^{j}$. By an entirely similar recursion, this time around applying $F$ to the powers of $Y=\exp (X)$, one sees that the coefficients $h_{j}$ belong to $A$.

We will set up an inductive procedure to show that these coefficients vanish.

For any $m \geq 1$, write $Y_{m}=\exp (X)-\sum_{i=1}^{m} X^{i} / i !=X^{m+1} /(m+1) !+\cdots$ and $f_{m}^{(2)}=Y_{m}^{2} / X^{m}$. Clearly, $f_{m}^{(2)} \in k[[X]] \cap k(X, \exp (X))=A^{\prime}$. Moreover, $\partial_{Y}\left(f_{m}^{(2)}\right)=$ $2 Y_{m} / X^{m}$ since $\partial_{Y}\left(Y_{m}\right)=\partial_{Y}(Y)=1$. By definition, $f_{m}^{(2)} \in A$, hence $F\left(f_{m}^{(2)}\right) \in$ $A$ by the assumption of item (b). Since $F\left(f_{m}^{(2)}\right)=2 h_{1} Y_{m} / X^{m}+2 h_{2} / X^{m}$ and $h_{1} Y_{m} / X^{m} \in A^{\prime}$ (as $h_{1} \in A \subset A^{\prime}$ ), it follows that $h_{2} / X^{m} \in A^{\prime}$. Since this holds for all $m \geq 1, h_{2} \in \bigcap_{m \geq 1} A^{\prime} X^{m}=0$. This shows that at least $h_{2}$ vanishes and also that $h_{1} Y_{m} / X^{m} \in A$. Again, by definition of $A, \partial_{Y}\left(h_{1} Y_{m} / X^{m}\right) \in A^{\prime}$. But $\partial_{Y}\left(h_{1} Y_{m} / X^{m}\right)=\partial_{Y}\left(h_{1}\right) Y_{m} / X^{m}+h_{1} / X^{m}$ and $\partial_{Y}\left(h_{1}\right) Y_{m} / X^{m} \in A^{\prime}$ (as $h_{1} \in A \Rightarrow \partial_{Y}\left(h_{1}\right) \in A^{\prime}$ and, as shown above, $\left.Y_{m} / X^{m} \in A^{\prime}\right)$. It follows that $h_{1} / X^{m} \in A^{\prime}$; hence $h_{1}=0$ as $m \geq 1$ is arbitrarily large.

Now assume that $i \geq 3$ and that $h_{1}=\ldots=h_{i-1}=0$ by the inductive assumption. For any $m \geq 1$, write $g_{m}^{(i)}=Y_{m}^{i} / X^{m}$. As before, $g_{m}^{(i)}$ belongs to $A^{\prime}$ and so does $\partial_{Y}\left(g_{m}^{(i)}\right)=i Y_{m}^{i-1} / X^{m}$; hence $g_{m}^{(i)} \in A$ and, by the standing assumption of this item, $F\left(g_{m}^{(i)}\right) \in A$. But $\partial_{Y}^{i}\left(g_{m}^{(i)}\right)=i ! h_{i} / X^{m}$ and $\partial_{Y}^{i+1}\left(g_{m}^{(i)}\right)=0$. Therefore, $i ! h_{i} / X^{m}=F\left(g_{m}^{(i)}\right) \in A$. Since $m \geq 1$ is arbitrary, $h_{i}=0$ as was to be shown.

Proof of Proposition (1.2). Recall the notation $\Delta=\left.d\right|_{A}$. In order to see that the subset $\left\{\Delta, \ldots, \Delta^{n}\right\} \subset \operatorname{Der}_{k}^{n}(A)$ is linearly independent over $A$, let $a_{1} \Delta+\cdots+$ $a_{n} \Delta^{n}=0$ be an $A$-linear relation. Evaluating both sides of this equality successively on the powers of $X$ yields $a_{1}=\ldots=a_{n}=0$.

We now show that $\operatorname{Der}_{k}^{n}(A)=\sum_{i=1}^{n} A \Delta^{i}$. By [8, Theorem 15], every element of $\operatorname{Der}_{k}^{n}(A)$ extends uniquely to an element of $\operatorname{Der}_{k}^{n}(k(X, Y))=\operatorname{der}_{k}^{n}(k(X, Y))$. Therefore, there is a natural identification

$$
\operatorname{Der}_{k}^{n}(A)=\left\{D \in \operatorname{Der}_{k}^{n}(k(X, Y)) \mid D(A) \subset A\right\} .
$$


Thus, let $D$ be an element in the right side of the above equality. By Lemma (1.4), one can write $D=E+F$, with $E \in \sum_{i=1}^{n} A d^{i}$ and $F(A) \subset A$. To conclude the proof, it suffices to show that $F=0$. Decompose $F=F_{0}+\cdots+F_{n-1}$, with $F_{i}=\sum_{1 \leq j \leq n-i} h_{i j} d^{i} \circ \partial_{Y}^{j}$ and $h_{i j} \in k(X, Y)$. Write

$$
G_{n-1}=[\ldots[F, \underbrace{X], \ldots, X}_{n-1 \text { times }}] .
$$

Since $F(A) \subset A$ and $X \in A$ then $G_{n-1}(A) \subset A$ as well. By Lemma (1.3), $G_{n-1}=$ $(n-1) ! h_{n-1,1} \partial_{Y}$ holds in $A^{\prime}$. Then, by Lemma (1.4)(b), $G_{n-1}=0$. Therefore, $h_{n-1,1}=0$ and, consequently, $F_{n-1}=h_{n-1,1} d^{n-1} \circ \partial_{Y}=0$.

By descending induction, assume that $F_{n-1}=\ldots=F_{i+1}=0$. Write

$$
G_{i}=[\ldots[F, \underbrace{X], \ldots, X}_{i \text { times }}] .
$$

By the same token as above, $G_{i}(A) \subset A$, and by Lemma (1.3),

$$
G_{i}=i ! \sum_{1 \leq j \leq n-i} h_{i j} \partial_{Y}^{j}
$$

By Lemma (1.4)(b), $G_{i}=0$, consequently $F_{i}=0$. This shows that $F=0$ as sought and concludes the proof of the theorem.

\section{THE SECOND EXAMPLE}

Recall that a Noetherian domain $A$ is said to be pseudo-geometric (or a Nagata domain) if for every prime ideal $\wp \subset A$ and every finite field extension $L$ of the field of fractions of $A / \wp$, the integral closure of $A / \wp$ in $L$ is a finite $A / \wp$-module.

The ring given in the previous section is not pseudo-geometric as its integral closure is not a finite module. In this section we give an example of a one-dimensional pseudo-geometric local domain $A$, admitting a coefficient field of characteristic zero, such that $A$ is not integrally closed and $\operatorname{der}_{k}(A)=0$. Thus, the Zariski-Lipman conjecture fails to be true in this class of local domains. Moreover, we will show that the Nakai conjecture fails as well in the same class of local domains.

The simplest such example $A$ admitting a coefficient field $k$ could in principle be such that its integral closure $A^{\prime}$ is a finite free $A$-module of rank 2. Moreover, since $A$ had better not be essentially of finite type over $k$, likewise its field of fractions may be allowed to have a transcendence degree over $k$ which is $\geq 2$. With these guiding principles in mind, we proceed to give one such explicit example.

As preliminaries, let $k$ be a field of characteristic zero and, as in the previous section, let $d_{X} \in \operatorname{der}_{k}(k[[X]])$ be the derivation sending $X^{i}$ to $i X^{i-1}$. Let $\pi \in$ $X^{2} k[[X]]$ be an element transcendental over $k(X)$ such that $d_{X}(\pi) \notin k(X, \pi)$, e.g., $\pi=\exp (\exp (X)-1)-X-1$ will do (cf. [1). In particular, the subfield $k(X, \pi) \subset$ $k((X))$ is a purely transcendental extension of $k$ of transcendence degree 2 . Set $A=\left(k+X^{2} k[[X]]\right) \cap k(X, \pi) \subset B=k[[X]] \cap k(X, \pi)$.

(2.1) Proposition. With the preceding setting, one has:

(i) The field of fractions of $A$ is $k(X, \pi)$ and the integral closure of $A$ is $B$.

(ii) $A$ is a one-dimensional pseudo-geometric local domain $A$.

(iii) $\operatorname{der}_{k}(A)=0$. 
Proof. (i) Since $X=X^{3} / X^{2}$, while $X^{2}, X^{3} \in A$ and $\pi \in A$, the first assertion is clear. For the second assertion, note that $B$ is integrally closed as it is a valuation ring. Moreover, clearly, $B=A+A X$, hence $\{1, X\}$ is a set of generators of $B$, showing that $B$ is a finite $A$-module. Combining these data, the second assertion is clear.

(ii) Since $B$ is a discrete valuation ring integral over $A$ then $A$ has dimension one. Furthermore, any one-dimensional domain containing a perfect field $k$ is pseudogeometric.

(iii) It is well known that for a domain $A$ containing a field of characteristic zero, whose integral closure is a finite $A$-module, every (ordinary) derivation of $A$ extends to a derivation of the integral closure (cf. [10, $\S 3$, Theorem]). Therefore, it suffices to show that $\operatorname{der}_{k}(B)=0$. Supposing otherwise, one then has $\operatorname{rkder}_{k}(B) \geq 1$, hence $\operatorname{rk} \operatorname{der}_{k}(B)=1$ since $\operatorname{dim} B=1$ (cf. 6, Theorem 98]). Then $d_{X}(B) \subset B$ by [6, Theorem 99], and therefore $d_{X}(\pi) \in B \subset k(X, \pi)$, a contradiction.

(2.2) Remark. As a side comment, we note that $B$ is a rank one discrete valuation ring (hence, regular) whose module $\Omega_{k}(B)$ of Kähler differentials is not free. Indeed, the rank of $\Omega_{k}(B)$ as $B$-module is the rank of its generic fiber $\Omega_{k}(B) \otimes_{B} K$ over $K=k(X, \pi)$. But the latter is 2 since $K$ has transcendence degree 2 over $k$. If $\Omega_{k}(B)$ were $B$-free it would then have a free $B$-basis with 2 elements which is impossible because its $B$-dual $\operatorname{der}_{k}(B)=0$. Of course, behind the scenes is the fact that $\Omega_{k}(B)$ is an infinitely generated $B$-module.

(2.3) Corollary. Let $A$ be as in Proposition (2.1). Then Zariski-Lipman and Nakai conjectures are both false for A.

Proof. By Proposition (2.1), $\operatorname{der}_{k}(A)=0$, certainly a free $A$-module, while $A$ is not regular. Therefore, Zariski-Lipman conjecture fails here. For the Nakai conjecture, one shows that the vanishing of the ordinary derivations implies the vanishing of all high order derivations, consequently the equalities $\operatorname{Der}_{k}^{n}(A)=\operatorname{der}_{k}^{n}(A)$ hold trivially for every $n$ (note that one cannot use the result of [9] as the proof there uses reduction to the normal case since the setting is that of rings essentially of finite type over a field). We choose to isolate this simple general result as a lemma.

(2.4) Lemma. Let $A$ be a commutative ring and let $k \subset A$ be a subring. If $\operatorname{der}_{k}(A)=0$, then $\operatorname{Der}_{k}^{n}(A)=0$ for every $n \geq 0$.

Proof. One proceeds by induction on $n$. The assumption implies the case $n=1$, so let $\operatorname{Der}_{k}^{i}(A)=\operatorname{der}_{k}^{i}(A)=0$ for $i \leq n-1$. Let $a \in A$ and $D \in \operatorname{Der}_{k}^{n}(A)$ be arbitrary. Then $[D, a] \in \operatorname{Diff}_{k}^{n-1}(A)=A \oplus \operatorname{Der}_{k}^{n-1}(A)=A$, by definition and by the inductive hypothesis, respectively. It follows that $[[D, a], b]=0$ for every $b \in A$. Once more, by definition, this means that $D \in \operatorname{Diff}_{k}^{1}(A)=A \oplus \operatorname{Der}_{k}^{1}(A)=A$. Since $D \in \operatorname{Der}_{k}^{n}(A), D$ is just multiplication by $D(1)=0$.

\section{REFERENCES}

1. J. Ax, On Schanuel's conjectures, Ann. of Math. 93 (1971), 252-268. MR 43:3215

2. K. R. Goodearl and T. H. Lenagan, Constructing bad Noetherians domains using derivations, J. Algebra 123 (1989), 478-495. MR 90g:13011

3. A. Grothendieck, Éléments de Géométrie Algèbrique, $4^{\text {ième }}$ Partie, IHES, Publications Mathématiques, 1967.

4. J. Lipman, Free derivations modules, Amer. J. Math. 87 (1965), 875-898. MR 32:4130 
5. A. K. Maloo, Differential simplicity and the module of derivations, J. Pure Appl. Algebra 115 (1997), 81-85.

6. H. Matsumura, Commutative Algebra, 2nd edition, Benjamin/Cummings, 1980. MR 82i: 13003

7. K.R. Mount and O.E. Villamayor, On a conjecture of Y. Nakai, Osaka J. Math. 10 (1973), 325-327. MR 48:6073

8. Y. Nakai, High order derivations I, Osaka J. Math. 7 (1970), 1-27. MR 41:8404

9. C. J. Rego, Remarks on differential operators on algebraic varieties, Osaka J. Math. 14 (1977), 481-486. MR [58:22060]

10. A. Seidenberg, Derivations and integral closure, Pacific J. Math 16 (1966), 167-173. MR 32:5686

11. W. Traves, Nakai's conjecture for varieties smoothed by normalization, Proc. Amer. Math. Soc. 127 (8) (1999), 2245-2248. MR 99j:13021

IMECC-UNICAMP, 13081-970 CAMPINAS, SÃo PAULO, BrazIL

E-mail address: brumatti@ime.unicamp.br

Instituto de Matemática Pura e Aplicada, Estrada Dona Castorina 110, J. Botânico, 22460-320, Rio DE JANeiro, RJ, BraziL

E-mail address: ylequain@impa.br

Instituto de Ciências Matemáticas e de Computação, USP-SC, Av. Dr. Carlos Botelho, 1465, 13560-970 SÃo Carlos, SP, Brazil

E-mail address: lev@icmsc.sc.usp.br

Departamento de Matemática, CCen, Universidade Federal de Pernambuco, 50740540 RECIFE, PE, BRAZIL

E-mail address: aron@dmat.ufpe.br 